\title{
Multiple Target Repetitive Transcranial Magnetic Stimulation (rTMS) Combined with Neurofeedback for Complete Resolution of Severe OCD, Bipolar Depression, and Anxiety
}

\author{
Ali Elahi* \\ Neurospa Brain Rejuvenation Center Inc., California, USA \\ ${ }^{\star}$ Corresponding author: Ali Elahi M.D, Neurospa Brain Rejuvenation Center Inc., California, USA
}

Received: August 03, 2021; Accepted: August 10, 2021; Published: August 20, 2021

Obsessive compulsive disorder (OCD) is a prevalent disabling condition and often comorbid with depression, anxiety, and high suicide rates. First line treatments of OCD such as Selective Serotonin Reuptake Inhibitors (SSRIs) and dopamine antagonists, and mood stabilizers fail to treat OCD in up to $60 \%$ of patients [1]. Transcranial magnetic stimulation has emerged as a non-pharmacological alternative for treatment of OCD and several case reports and randomized trials have shown positive response although the efficacy of $\mathrm{r}$ TMS remains low at approximately $35 \%$ with little positive effects on reversing comorbities including symptoms of depression and anxiety [2]. The most effective stimulation parameters, cortical targets, and type of coil (figure of eight, H-coil, cone, or deep) for rTMS have not been established. Investigators report positive outcomes with stimulation of the right Orbitofrontal Cortex (OFC), Supplementary Motor Area (SMA), and Anterior Cingulate Cortex (ACC) [2,3]. Recently, deep $\mathrm{r}$ tms directed at ACC has show significant benefit [4]. In addition, neurofeedback, also known as 'EEG biofeedback', has been established as an effective treatment of various psychological and neuropsychiatric disorders including OCD, anxiety, and depression $[5,6]$. Accordingly, I hypothesize that a more formidable outcome can be achieved in patients suffering from OCD accompanied by comorbidities when: 1) multiple cortical targets are stimulated under one treatment regimen; 2) multiple stimulation parameters are utilized in response to patient report of outcome; and 3) neurofeedback is used in conjunction with rTMS. This 'combination approach' was in fact, found to be significantly effective in treatment of pervasive spectrum disorder, e.g. autism and early signs of dementia [7,8]. Here, I present the first case report of patient with severe OCD, anxiety, and depression who showed marked improvement and resolution of OCD, anxiety, and depression following completing total of 41 sessions of rTMS and 15 sessions of ' $Z$-score neurofeedback'.

Patient is 33-year-old male diagnosed with over 15 years of OCD, generalized anxiety, and bipolar depression. Patient has undergone trials of Serotonin Reuptake Inhibitors (SSRIs), mood stabilizers including lithium and valproic acid, and benzodiazepines including lorazepam. At time of presentation to my clinic, patient's primary symptoms included obsessive thoughts with facial and skin contamination, recurrent feeling of self-guilt, marked difficulty with anxiety in dark environments, agoraphobia, poor self-worth, and extreme spells of depression, manic, and hypomanic episodes. In addition, patient displayed severe hypochondriasis with respect to various disorders including motor neuron disease, multiple sclerosis, and infections. Moreover, patient experienced various somatic symptoms including non-specific vibratory sensations of mouth, trunk, and extremities. At onset, Yale Brown Obsessive Compulsive Score (Y-BOCS) was 19, Burn's anxiety score was 36, Burn's depression score was 22, and PHQ-9 of 10. Risks and benefits of rTMS including off-label use of rTMS parameters were discussed in detail with patient and written signed consent obtained. A brain MRI was obtained to include skin fiduciary markers for navigation software (The Neural Navigator, Brain Science Tools, Utrechet, Netherlands) 1mm sagittal and axial T1 MRI images were processed and segmented to identify several cortical targets including: left and right Dorsolateral Prefrontal Cortex (DLPFC), right OFC, left Dorsomedial Prefrontal Cortex (DMPFC), and ACC. On initial visit and every 5-6 visits, the Motor Threshold (MT) was determined as the intensity required to active the left Abductor Policis Brevis (APB) and/or the First Dorsal Interosseus (FDI) on the contralateral hand on average of 5 of 10 single pulse trials directed at left motor cortex per visual inspection. Patient was interviewed and underwent daily rTMS sessions using varying targets and stimulation parameters (see table). The rTMS stimulation parameters were chosen based on current trial evidence for treatment of OCD, depression, and anxiety and patient's daily report of signs and symptoms. All stimulations were performed using figure-of-eight coil (Neurosoft, Ltd. Ivanovo, Russian Federation) rTMS machine. Cortical targets included SMA, left DLPFC, right DLPFC, left DMPFC, ACC, and right OFC. Cortical targets were identified and marked using navigation software.

Neurofeedback was done using Neuroguide 'Z-Score LORETA' neurofeedback software by collecting and editing 4 minutes of baseline EEG and creating a 'symptoms checklist match' (for detail of procedure see Thatcher \& Lubar, Z Score Neurofeedback: Clinical 


\begin{tabular}{|c|c|c|}
\hline \multicolumn{2}{|c|}{ Treatment Date } & Location of Stimulation \\
\hline \multicolumn{3}{|c|}{1 7/9/2018 SMA } \\
\hline \multicolumn{3}{|c|}{$27 / 10 / 2018$ SMA } \\
\hline \multicolumn{3}{|c|}{3 7/11/2018 SMA } \\
\hline \multicolumn{3}{|c|}{4 7/12/2018 SMA } \\
\hline \multicolumn{3}{|c|}{5 7/13/2018 SMA } \\
\hline \multicolumn{3}{|c|}{6 7/16/2018 LDLPFC } \\
\hline \multicolumn{3}{|c|}{7 7/17/2018 LDLPFC } \\
\hline \multicolumn{3}{|c|}{8 7/18/2018 LDLPFC } \\
\hline & \multicolumn{2}{|c|}{ 7/19/2018 LDLPFC } \\
\hline 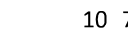 & \multicolumn{2}{|c|}{0 7/20/2018 LDLPFC/RDLPFC } \\
\hline 117 & \multicolumn{2}{|c|}{1 7/23/2018 RDLPFC } \\
\hline 127 & \multicolumn{2}{|c|}{$7 / 24 / 2018$ RDLPFC } \\
\hline 137 & \multicolumn{2}{|c|}{ 7/25/2018 RDLPFC } \\
\hline 147 & \multicolumn{2}{|c|}{ 7/26/2018 LDLPFC/RDLPFC } \\
\hline 157 & \multicolumn{2}{|c|}{ 7/27/2018 LDLPFC/SMA } \\
\hline 167 & \multicolumn{2}{|c|}{ 7/30/2018 LDLPFC/SMA } \\
\hline 177 & \multicolumn{2}{|c|}{ 7/31/2018 LDLPFC/SMA } \\
\hline 18 & \multicolumn{2}{|c|}{ 8/1/2018 LDLPFC/SMA } \\
\hline 19 & \multicolumn{2}{|c|}{ 8/2/2018 LDLPFC/SMA } \\
\hline 20 & \multicolumn{2}{|c|}{$8 / 3 / 2018$ RDLPFC } \\
\hline 21 & \multicolumn{2}{|c|}{ 8/9/2018 LDLPFC/SMA } \\
\hline 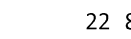 & \multicolumn{2}{|c|}{ 8/10/2018 LDLPFC/SMA } \\
\hline $23 \varepsilon$ & $8 / 13 / 2018$ & 3 LDLPFC/SMA \\
\hline 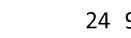 & $9 / 10 / 2018$ & 3 LDLPFC/RDLPFC/ACC/OFC \\
\hline 25 & 9/11/2018 & 3 LDLPFC/RDLPFC/ACC/OFC \\
\hline $26 s$ & 9/12/2018 & 3 RDLPFC \\
\hline $27 \mathrm{~s}$ & 9/13/2018 & 3 RDLPFC \\
\hline 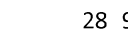 & 9/14/2018 & 3 RDLPFC \\
\hline 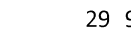 & 9/17/2018 & 3 LDLPFC/RDLPFC/ACC/OFC \\
\hline $30 \mathrm{~s}-\mathrm{s}-\mathrm{r}$ & 9/19/2018 & 3 LDLPFC/RDLPFC/ACC/OFC \\
\hline $31 \mathrm{c}$ & 9/20/2018 & 3 LDLPFC/RDLPFC/ACC/OFC \\
\hline 32 & 9/21/2018 & 3 LDLPFC/RDLPFC/ACC/OFC \\
\hline $33 \mathrm{c}$ & 9/24/2018 & 3 LDLPFC/RDLPFC/ACC/OFC/LDMPFC \\
\hline $34 \mathrm{~s}$ & 9/25/2018 & 3 LDLPFC/RDLPFC/ACC/OFC/LDMPFC \\
\hline $35 \mathrm{c}$ & $9 / 26 / 2018$ & 3 LDLPFC/RDLPFC/ACC/OFC/LDMPFC \\
\hline $36 s$ & 9/27/2018 & 3 LDLPFC/RDLPFC/ACC/OFC/LDMPFC \\
\hline 37 & $10 / 2 / 2018$ & 3 LDLPFC/RDLPFC/ACC/OFC/LDMPFC \\
\hline 38 & $10 / 3 / 2018$ & 3 LDLPFC/RDLPFC/ACC/OFC/LDMPFC \\
\hline 39 & $10 / 4 / 2018$ & 3 LDLPFC/RDLPFC/ACC/OFC/LDMPFC \\
\hline 401 & $10 / 5 / 2018$ & 3 LDLPFC/RDLPFC/ACC/OFC/LDMPFC \\
\hline 41 & $10 / 8 / 2018$ & 3 LDLPFC/RDLPFC/ACC/OFC/LDMPFC \\
\hline & Legend & SMA \\
\hline & & LDLPFC \\
\hline & & RDLPFC \\
\hline & & $\mathrm{ACC}$ \\
\hline & & OFC \\
\hline & & LDMPFC \\
\hline & & іTBS \\
\hline & & cTBS \\
\hline
\end{tabular}

\begin{tabular}{|c|c|c|c|c|c|}
\hline Frequency $(\mathrm{Hz})$ & Inter-train interval (sec) & Treatment Intensity (\% of MT) & Pulses in Train & No. of Trains & MT \\
\hline $1 \mathrm{~Hz}$ & 60 & $100 \%$ & 300 & 4 & 57 \\
\hline $1 \mathrm{~Hz}$ & 60 & $100 \%$ & 300 & 4 & 57 \\
\hline $1 \mathrm{~Hz}$ & 60 & $100 \%$ & 300 & 4 & 57 \\
\hline $1 \mathrm{~Hz}$ & 60 & $100 \%$ & 300 & 4 & 57 \\
\hline $1 \mathrm{~Hz}$ & 60 & $100 \%$ & 300 & 4 & 57 \\
\hline iTBS & 10 & $80 \%$ & 30 & 60 & 60 \\
\hline iTBS & 10 & $80 \%$ & 30 & 60 & 60 \\
\hline iTBS & 10 & $80 \%$ & 30 & 60 & 60 \\
\hline iTBS & 10 & $80 \%$ & 30 & 60 & 60 \\
\hline iTBS/CTBS & $10-1$ & $80--80 \%$ & $30 / 300$ & $60--6$ & $60 / 65$ \\
\hline $1 \mathrm{~Hz}$ & 1 & $80 \%$ & 20 & 45 & 60 \\
\hline $1 \mathrm{~Hz}$ & 1 & $80 \%$ & 20 & 45 & 60 \\
\hline $1 \mathrm{~Hz}$ & 1 & $80 \%$ & 20 & 45 & 60 \\
\hline iTBS/CTBS & $10--1$ & $80--80 \%$ & $30 / 300$ & $60--6$ & $60 / 60$ \\
\hline iTBS/1 Hz & $10-60$ & $80--100 \%$ & $30 / 300$ & $60-4$ & $60 / 60$ \\
\hline iTBS/1 Hz & $10--60$ & $80-100 \%$ & $30 / 300$ & $60-4$ & $60 / 60$ \\
\hline iTBS/1 Hz & $10--60$ & $80--100 \%$ & $30 / 300$ & $60-4$ & $60 / 60$ \\
\hline iTBS/1 Hz & $10--60$ & $80-100 \%$ & $30 / 300$ & $60-4$ & $60 / 60$ \\
\hline iTBS/1 Hz & $10--60$ & $80--100 \%$ & $30 / 300$ & $60-4$ & $60 / 60$ \\
\hline cTBS & 0 & $80 \%$ & 1800 & 1 & 60 \\
\hline iTBS/1 Hz & $10--60$ & $80 / 100 \%$ & $30 / 300$ & $60-4$ & $60 / 60$ \\
\hline iTBS/1 Hz & $10--60$ & $80 / 100 \%$ & $30 / 300$ & $60-4$ & $60 / 60$ \\
\hline iTBS/1 Hz & $10--60$ & $80 / 100 \%$ & $30 / 300$ & 60--4 & $60 / 60$ \\
\hline $10 / 10 / 10 / 1$ & $26 / 26 / 26 / 0$ & $120 / 120 / 140 / 100 \%$ & $40 / 40 / 40 / 300$ & $41 / 41 / 25 / 4$ & $55 / 57$ \\
\hline $10 / 10 / 10 / 1$ & $26 / 26 / 26 / 0$ & $120 / 120 / 140 / 100 \%$ & $40 / 40 / 40 / 300$ & $41 / 41 / 25 / 4$ & $55 / 57$ \\
\hline 10 & 26 & $120 \%$ & 40 & 125 & 57 \\
\hline 10 & 26 & $120 \%$ & 40 & 125 & 57 \\
\hline 10 & 26 & $130 \%$ & 40 & 125 & 57 \\
\hline $10 / 10 / 10 / 1$ & $26 / 26 / 26 / 0$ & $120 / 120 / 130 / 130 \%$ & $40 / 40 / 40 / 300$ & $41 / 41 / 25 / 4$ & $55 / 57$ \\
\hline $10 / 10 / 10 / 1$ & $26 / 26 / 26 / 0$ & $110 / 120 / 140 / 120 \%$ & $40 / 40 / 40 / 300$ & $41 / 41 / 25 / 4$ & $55 / 57$ \\
\hline $10 / 10 / 10 / 1$ & $26 / 26 / 26 / 0$ & $120 / 110 / 140 / 120 \%$ & $40 / 40 / 40 / 300$ & $41 / 41 / 25 / 4$ & $55 / 57$ \\
\hline 10/10/10/1 & $26 / 26 / 26 / 0$ & $120 / 110 / 140 / 120 \%$ & $40 / 40 / 40 / 300$ & $41 / 41 / 25 / 4$ & $55 / 57$ \\
\hline 10/10/10/1/iTBS & $26 / 26 / 26 / 0 / 10$ & $120 / 120 / 140 / 120 / 100 \%$ & $40 / 40 / 40 / 300 / 30$ & $41 / 41 / 25 / 4 / 20$ & $55 / 57$ \\
\hline 10/10/10/1/iTBS & $26 / 26 / 26 / 0 / 10$ & $120 / 120 / 140 / 120 / 100 \%$ & $40 / 40 / 40 / 300 / 30$ & $41 / 41 / 25 / 4 / 20$ & $55 / 57$ \\
\hline 10/10/10/1/iTBS & $26 / 26 / 26 / 0 / 10$ & $120 / 120 / 140 / 120 / 100 \%$ & $40 / 40 / 40 / 300 / 30$ & $41 / 41 / 25 / 4 / 20$ & $55 / 57$ \\
\hline 10/10/10/1/iTBS & $26 / 26 / 26 / 0 / 10$ & $120 / 120 / 140 / 120 / 100 \%$ & $40 / 40 / 40 / 300 / 30$ & $41 / 41 / 25 / 4 / 20$ & $55 / 57$ \\
\hline 10/10/10/1/iTBS & $26 / 26 / 26 / 0 / 10$ & $120 / 120 / 140 / 120 / 100 \%$ & $40 / 40 / 40 / 300 / 30$ & $41 / 41 / 25 / 4 / 20$ & $55 / 57$ \\
\hline 10/10/10/1/iTBS & $26 / 26 / 26 / 0 / 10$ & $120 / 120 / 140 / 120 / 100 \%$ & $40 / 40 / 40 / 300 / 30$ & $41 / 41 / 25 / 4 / 20$ & $55 / 57$ \\
\hline 10/10/10/1/iTBS & $26 / 26 / 26 / 0 / 10$ & $120 / 120 / 140 / 120 / 100 \%$ & $40 / 40 / 40 / 300 / 30$ & $41 / 41 / 25 / 4 / 20$ & $55 / 57$ \\
\hline 10/10/10/1/iTBS & $26 / 26 / 26 / 0 / 10$ & $120 / 120 / 140 / 120 / 100 \%$ & $40 / 40 / 40 / 300 / 30$ & $41 / 41 / 25 / 4 / 20$ & $55 / 57$ \\
\hline 10/10/10/1/iTBS & $26 / 26 / 26 / 0 / 10$ & $120 / 120 / 140 / 120 / 100 \%$ & $40 / 40 / 40 / 300 / 30$ & $41 / 41 / 25 / 4 / 20$ & $55 / 57$ \\
\hline
\end{tabular}

supplementary motor area

left dorsolateral prefrontal cortex right dorsolateral prefrontal cortex anterior cingulate cortex

right orbital frontal cortex

left dorsomedial prefrontal cortex

intermittent theta burst

continuous theta burst
Total Pulses 
Applications, 2015; Thatcher, Latest Developments in Live Z-Score Training: Symptom Check List, Phase Reset, and Loreta Z-Score Biofeedback, 2013) $[9,10]$. Each session consistent of five 5-minute rounds for total of approximately 25-30 minutes. Dry, wireless, headset (DSI-24, Wearable Sensing, San Diego, CA, USA) was used to gather EEG recordings and conduct neurofeedback sessions. Patient underwent neurofeedback approximately two times per week, usually prior to or after $r$ TMS sessions. Patient showed progressive and marked improvement in, OCD, anxiety, and depression. Y-BOCS scores showed 63\% improvement (19 to 7), Burn's depression showed $86 \%$ improvement (22 to 3 ), Burn's anxiety showed $72 \%$ improvement (36 to 10), and PHQ-9 showed 70\% improvement (from 10 to 3 ). Patient reported complete reversal of agoraphobia, self-contamination delusions, and depression. He reported feeling quite comfortable with going to large dark movie theater, avoiding washing rituals, denied panic attacks in provocative environments (e.g. driving), and developed markedly improved mood. The clinical improvements were noticeable by father and other close relatives. Although we delivered higher intensity of stimulation and overall larger total daily pulses, patient reported no significant adverse effects and did not experience seizures. To our knowledge, this is first report of 1) applying rTMS to multiple (e.g. more than 2) cortical targets on one patient with each utilizing separate stimulation protocols, and 2) adding neurofeedback to treatment regimen leading to marked improvement and resolution of OCD together with anxiety and depression.

The rationale behind use of various cortical targets is to modulate several circuits that may be contributing to OCD and comorbidities including abnormal connectivity and/or neuronal hyperactivity within cortical-striate-thalamic-cortical circuits--currently the leading working model regarding the pathophysiology of OCD [4]. One randomized, sham-controlled study showed modest improvements in OCD (29\% reduction on Y-BOCS) and depression (48\% on HAM-D) symptoms after 14 sequential $1 \mathrm{~Hz}$ stimluation sessions targeting left DLPFC and SMA [11,12]. Additional randomized sham-controlled studies applying a multi-target approach and in combination with neurofeedback will be helpful and can potentially significanly increase the efficacy rate of treatment.

Keywords: Generalized anxiety disorder, Multiple targets $r$ TMS, Neurofeedback, Obsessive compulsive disorder, Repetitive transcranial magnetic stimulation

\section{References}

1. Pallanti S, Quercioli L (2006) Treatment-refractory obsessive-compulsive disorder: Methodological issues, operational definitions and therapeutic lines. Progress in Neuro-psychopharmacology \& Biological Psychiatry 30: 400-412. [crossref]

2. Berlim MT, Neufeld NH, Van den Eynde F (2013) Repetitive transcranial magnetic stimulation (rTMS) for obsessive-compulsive disorder (OCD): An exploratory meta-analysis of randomized and sham-controlled trials. Journal of Psychiatric Research 47: 999-1006. [crossref]

3. Nauczyciel C, Le Jeune F, Naudet F, Douabin S, Esquevin A, et al. (2014) Repetitive transcranial magnetic stimulation over the orbitofrontal cortex for obsessivecompulsive disorder: a double-blind, crossover study. Translational Psychiatry 4: e436. [crossref]

4. Blom RM, Figee M, Vulink N, Denys D (2011) Update on Repetitive Transcranial Magnetic Stimulation in Obsessive-Compulsive Disorder: Different Targets. Current Psychiatry Reports 13: 289-294. [crossref]

5. Sürmeli T, Ertem A (2011) Obsessive Compulsive Disorder and the Efficacy of qEEG-
Guided Neurofeedback Treatment: A Case Series. Clinical Eeg and Neuroscience 42: 195-201. [crossref]

6. Hammond DC (2003) QEEG-Guided Neurofeedback in the Treatment of Obsessive Compulsive Disorder. Journal of Neurotherapy 7: 25-52.

7. Sokhadze EM, El-Baz AS, Tasman A, Sears LL, Wang Y, et al. (2014) Neuromodulation integrating rTMS and neurofeedback for the treatment of autism spectrum disorder: an exploratory study. Applied Psychophysiology and Biofeedback 39: 237-257. [crossref]

8. Rabey JM, Dobronevsky E, Aichenbaum S, Gonen O, Marton RG, et al. (2013) Repetitive transcranial magnetic stimulation combined combined with cognitive training is a safe and effective modality for treatment of Alzheimer's diseas: a randomizeed double blind study. J Neural Transm 120: 813-819. [crossref]

9. Thatcher RW (2013) Latest Developments in Live Z-Score Training: Symptom Check List, Phase Reset, and Loreta Z-Score Biofeedback. Journal of Neurotherapy 17: 69-87.

10. Thatcher RW, Lubar JF (2015) Z Score Neurofeedback: Clinical Applications. Elsevier Inc.

11. Kang JI, Kim CH, Namkoong K, Lee CI, Kim SJ (2009) A randomized controlled study of sequentially applied transcranial magnetic stimulation obsessive compulsive disorder. J. Clin Psychiatry 70: 1645-51. [crossref]

12. Lubar JF (2015) Optimal Procedures in Z-Score Neurofeedback: Strategies for Maximizing Learning for Surface and LORETA Neurofeedback.

\section{Citation:}

Elahi A (2021) Multiple Target Repetitive Transcranial Magnetic Stimulation (rTMS) Combined with Neurofeedback for Complete Resolution of Severe OCD, Bipolar Depression, and Anxiety. J Neurol Neurocrit Care Volume 4(3): 1-3. 